\title{
CONVOLUTIONS WITH KERNELS HAVING SINGULARITIES ON A SPHERE
}

BY

\author{
ROBERT S. STRICHARTZ
}

\begin{abstract}
We prove that convolution with $\left(1-|x|^{2}\right)_{+}^{-\alpha}$ and related convolutions are bounded from $L^{p}$ to $L^{q}$ for certain values of $p$ and $q$. There is a unique choice of $p$ which maximizes the measure of smoothing $1 / p-1 / q$, in contrast with fractional integration where $1 / p-1 / q$ is constant. We apply the results to obtain a priori estimates for solutions of the wave equation in which we sacrifice one derivative but gain more smoothing than in Sobolev's inequality.
\end{abstract}

1. Introduction. For convolutions on Euclidean $n$-space $E_{n}$ with homogeneous kernels, the fractional integration theorem and the Calderón-Zygmund inequalities [3] give essentially best possible results. Such kernels have singularities at 0 and $\infty$. In this paper we study the boundedness in various $L^{p}$ norms of the convolution operator $T f(x)=\int_{E_{n}} f(x-y) K(y) d y$ for $K$ having its singularities on the unit sphere. The results are quite different in character from the case of homogeneous $K$. Such operators arise naturally in the study of the wave equation, and our results will give us new information about solutions of this equation.

More precisely, let $\Sigma$ denote the unit sphere in $E_{n}$, let $x, y$ denote points in $E_{n}$ and $x^{\prime}, y^{\prime}$ denote points in $\Sigma$. Let $d x^{\prime}$ be Lebesgue measure on $\Sigma$ normalized so that $\int_{E_{n}} f(x) d x=\int_{0}^{\infty} \int_{\Sigma} f\left(r x^{\prime}\right) d x^{\prime} r^{n-1} d r$. For each nonnegative integer and $f \in C_{\text {com }}^{\infty}$ we define

$$
T_{k+1} f(x)=\left.\int_{\Sigma}\left(\frac{\partial}{\partial r}\right)^{k} f\left(x-r y^{\prime}\right)\right|_{r=1} d y^{\prime}
$$

For $0<\alpha<1$ we define

$$
T_{\alpha} f(x)=\int_{|y| \leqq 1} f(x-y)\left(1-|y|^{2}\right)^{-\alpha} d y .
$$

For $\alpha \geqq 1$ the integral (2) no longer makes sense as such because $\left(1-|y|^{2}\right)^{-\alpha}$ has a nonintegrable singularity on $\Sigma$. However, we want to define $T_{\alpha}$ for nonintegral $\alpha$ in the range $0<\alpha \leqq(n+1) / 2$ by interpreting (2) in a distribution sense. We do this

Received by the editors May 15, 1969 and, in revised form, August 21, 1969.

AMS Subject Classifications. Primary 4425; Secondary 3576.

Key Words and Phrases. Convolution, $L^{p}$ estimate, spherical convolution, wave equation, energy estimate. 
by formally integrating by parts in the radial direction and neglect the boundary terms. Thus, if $k<\alpha<k+1$ we define

$$
T_{\alpha} f=\left(-\frac{1}{2}\right)^{k} \frac{\Gamma(\alpha-k)}{\Gamma(\alpha)} \int_{\Sigma} \int_{0}^{1}\left(1-r^{2}\right)^{k-\alpha}\left(\frac{\partial}{\partial r} \frac{1}{r}\right)^{k}\left(r^{n-1} f\left(x-r y^{\prime}\right)\right) d r d y^{\prime} .
$$

We can now state our main results:

THEOREM 1. (a) $\left\|T_{\alpha} f\right\|_{q} \leqq A_{\alpha}\|f\|_{p}$ provided $1<p \leqq 2 \leqq q<\infty$ and $1 / p-1 / q \leqq$ $(n+1-2 \alpha) / 2 n$ for $0<\alpha \leqq(n+1) / 2$.

(b) $\left\|T_{\alpha} f\right\|_{p^{\prime}} \leqq A_{\alpha}\|f\|_{p}$ for $p=(n+1) /(n+1-\alpha), p^{\prime}=(n+1) / \alpha$ and $0<\alpha \leqq(n+1) / 2$.

(c) $\left\|T_{\alpha} f\right\|_{q} \leqq A_{\alpha}\|f\|_{p}$ for $\frac{1}{2} \leqq \alpha \leqq(n+1) / 2$ provided $(n+1) /(n+1-\alpha) \leqq p \leqq 2$ and $n / q=\alpha-1 / p$, or $n /\left(n+\frac{1}{2}-\alpha\right) \leqq p \leqq(n+1) /(n+1-\alpha)$ and $1 / q=\alpha-n / p^{\prime}$.

We remark that the amount of smoothing in (b) is $1 / p-1 / p^{\prime}=(n+1-2 \alpha) /(n+1)$ which is more than in (a) provided $n \geqq 2$. On the other hand (a) is applicable to a wider range of $p$ 's. It is also possible to interpolate between these results in case $\alpha<\frac{1}{2}$. We leave the details to the interested reader.

In $\$ 2$ we prove Theorem 1 . In $\$ 3$ we given applications to the wave equation. In $\S 4$ we prove a similar result for spherical convolutions.

\section{Proof of Theorem 1 . Let}

$$
\begin{aligned}
\phi_{\alpha}(y) & =\left(1-|y|^{2}\right)^{-\alpha} & & \text { if }|y|<1, \\
& =0 & & \text { if }|y| \geqq 1 .
\end{aligned}
$$

If $\alpha<1, \phi_{\alpha} \in L^{1}$ so we can compute its Fourier transform. The result

$$
\hat{\phi}_{\alpha}(\xi)=(2 \pi)^{-n / 2} 2^{-\alpha} \Gamma(1-\alpha)|\xi|^{\alpha-n / 2} J_{n / 2-\alpha}(|\xi|)
$$

is well known [1] or [8]. We thus have

$$
T_{\alpha}(f)^{\wedge}(\xi)=(2 \pi)^{-n / 2} 2^{-\alpha} \Gamma(1-\alpha)|\xi|^{\alpha-n / 2} J_{n / 2-\alpha}(|\xi|) \hat{f}(\xi)
$$

for $0 \leqq \alpha<1$. But in fact (5) holds for all $\alpha \neq 1,2, \ldots$ (the poles of $\Gamma(1-\alpha)$ ). For the right-hand side of (5) is a single-valued analytic function of $\alpha$ in the complex plane minus the positive integers. On the other hand, the Fourier transform of the righthand side of (3), for fixed $k$, is also a single-valued analytic function of $\alpha$ in $\operatorname{Re}(\alpha)<k+1$ minus $\alpha=k, k-1, \ldots, 1$. These two functions agree for real $\alpha$ in the interval $0<\alpha<1$, hence by the principle of analytic continuation they are equal for $\alpha \neq 1,2, \ldots$

We can also compute $T_{k} f^{\wedge}(\xi)$ for $k=1,2, \ldots$ In fact $T_{k} f$ is the convolution of $f$ with the distribution $(\partial / \partial r)^{k} \sigma$, where $\sigma$ is Lebesgue measure on $\Sigma$, regarded as a finite measure in $E_{n}$, and $\partial / \partial r$ is radial differentiation. Now we have the wellknown formula [1]:

$$
\hat{\sigma}(\xi)=(2 \pi)^{n / 2}|\xi|^{1-n / 2} J_{n / 2-1}(|\xi|)
$$


Since $\partial / \partial r=(1 /|x|) x \cdot \nabla$ and $|x|=1$ on the support of $\sigma$, we have

$$
\left(\left(\frac{\partial}{\partial r}\right)^{k} \sigma\right)^{\wedge}(\xi)=(2 \pi)^{n / 2}\left(\sum_{j=1}^{n} \frac{\partial}{\partial \xi_{j}} \xi_{j}\right)^{k}\left(|\xi|^{1-n / 2} J_{n / 2-1}(|\xi|)\right) .
$$

Using the recursion relations [9]

$$
(d / d t)\left(t^{-s} J_{s}(t)\right)=-t^{-s} J_{s+1}(t)
$$

and

$$
J_{s+1}(t)=(2 s / t) J_{s}(t)-J_{s-1}(t)
$$

and the fact that

we easily obtain

$$
\sum_{j=1}^{n} \frac{\partial}{\partial \xi_{j}} \xi_{j}=n I d+|\xi| \frac{\partial}{\partial|\xi|}
$$

$$
\left(\left(\frac{\partial}{\partial r}\right)^{k} \sigma\right)^{\wedge}(\xi)=\sum_{i=1}^{k+1} c_{i}|\xi|^{i-n / 2} J_{n / 2-i}(|\xi|)
$$

for certain constants $c_{i}$ depending on $n$ and $k$. Thus we have

$$
T_{k} f^{\wedge}(\xi)=\sum_{i=1}^{k} c_{i}|\xi|^{i-n / 2} J_{n / 2-i}(|\xi|) \hat{f}(\xi) .
$$

We now define operators $S_{\alpha}$, for $\alpha$ in the strip $0 \leqq \operatorname{Re}(\alpha) \leqq(n+1) / 2$, and $f \in C_{\text {com }}^{\infty}$, by

$$
S_{\alpha} f^{\wedge}(\xi)=|\xi|^{\alpha-n / 2} J_{n / 2-\alpha}(|\xi|) \hat{f}(\xi) .
$$

It clearly suffices to prove estimates (a) (b) and (c) for $S_{\alpha}$ in place of $T_{\alpha}$.

We need the classical estimates on the size of the Bessel function [8, (11.10) and (11.11)],

$$
\left|t^{-(a+i b)} J_{a+i b}(t)\right| \leqq c_{a} e^{c|b|}(1+t)^{-a-1 / 2}
$$

for $0<t<\infty$. This is usually proved for $a>-\frac{1}{2}$ but it actually holds for all $a$ by (8). This estimate is sufficient to establish part (a) of the theorem as a consequence of the theorem of Hardy and Littlewood [3] that asserts that the operator $\mathscr{F}^{-1}(m(\xi) \hat{f}(\xi))$ is bounded from $L^{p}$ to $L^{q}$ provided $1<p \leqq 2 \leqq q<\infty$ and

$$
|m(\xi)| \leqq c|\xi|^{-t} \text { for } 1 / p-1 / q=t / n \text {. }
$$

For $S_{\alpha}$ has this form with $m(\xi)=|\xi|^{\alpha-n / 2} J_{n / 2-\alpha}(|\xi|)$.

Thus (12) implies (13) for any $t \leqq n / 2-\alpha+\frac{1}{2}$ which proves (a).

To prove (b) we use the fact that $S_{\alpha}$ is an analytic function of $\alpha$ and the interpolation theorem of Stein [7] for such analytic families of operators. We note that (12) restricts the growth of $\left\|S_{\alpha} f\right\|_{2}$ in the strip $0 \leqq \operatorname{Re}(\alpha) \leqq(n+1) / 2$ so that the theorem is applicable. On the boundary $\operatorname{Re}(\alpha)=(n+1) / 2$ we have $\left\|S_{\alpha} f\right\|_{2} \leqq$ 
$c \exp (c \operatorname{Im}(\alpha))\|f\|_{2}$ by (12) and the Plancherel theorem. On the boundary $\operatorname{Re}(\alpha)=0$ we use (5) and the obvious estimate $\left\|T_{\alpha} f\right\|_{\infty} \leqq\|f\|_{1}$ since $\left|\phi_{\alpha}(x)\right| \leqq 1$. Since $|\Gamma(1+i b)|=(\pi b / \sinh b)^{1 / 2}$ we obtain $\left\|S_{\alpha} f\right\|_{\infty} \leqq c \exp (c \operatorname{Im}(\alpha))\|f\|_{1}$ for $\operatorname{Re}(\alpha)=0$. Stein's theorem now implies part (b). We obtain (c) from (a) and (b) immediately by the M. Riesz interpolation theorem, if $\alpha>\frac{1}{2}$. For $\alpha=\frac{1}{2}$, we see that $T_{1 / 2}$ maps $L^{1}$ to weak $L^{2}$ since $\phi_{1 / 2}$ is in weak $L^{2}$. Applying the Marcinkiewicz interpolation theorem [10] and a duality argument [3] we obtain (c).

REMARKS. For $0 \leqq \alpha \leqq 1$ we can show that estimate (c) is sharp, in the sense that if $\left\|T_{\alpha} f\right\|_{q} \leqq M\|f\|_{p}$ then $1 / q \geqq(\alpha-1+1 / p) / n$. For if we choose $f$ to be $\phi_{\beta}$ it is not difficult to show that $\left|T_{\alpha} \phi_{\beta}(x)\right| \geqq c|x|^{-\beta-\alpha+1}$ for $|x| \leqq \frac{1}{2}$.

It should be possible to prove similar results for kernels obtained by replacing $|x|^{2}$ by any nondegenerate quadratic form by using the computations of $[1$, Chapter III, §2].

3. The wave equation. The Cauchy problem for the wave equation in $n$-space variables $x$ and time variable $t$

$$
\begin{gathered}
\partial^{2} u(x, t) / \partial t^{2}=\Delta_{x} u(x, t), \\
u(x, 0)=f(x), \quad \partial u(x, 0) / \partial t=g(x)
\end{gathered}
$$

for $f, g \in C_{\text {com }}^{\infty}\left(E_{n}\right)$ has a unique solution

$$
u(x, t)=\mathscr{F}^{-1}\left(\hat{f}(\xi) \cos t|\xi|+\hat{g}(\xi) \frac{\sin t|\xi|}{|\xi|}\right) .
$$

Now we have

$$
J_{1 / 2}(t)=\left(\frac{2}{\pi}\right)^{1 / 2} \frac{\sin t}{t^{1 / 2}} \text { and } \quad J_{-1 / 2}(t)=\left(\frac{2}{\pi}\right)^{1 / 2} \frac{\cos t}{t^{1 / 2}}
$$

so that (16) becomes

$$
u(x, t)=\left(\frac{\pi}{2}\right)^{1 / 2} \delta(t) S_{(n+1) / 2} \delta\left(t^{-1}\right) f+\left(\frac{\pi}{2}\right)^{1 / 2} t \delta(t) S_{(n-1) / 2} \delta\left(t^{-1}\right) g
$$

where $\delta(t)$ is the dilation operator

$$
\delta(t) f(x)=f(t x)
$$

We define the $p$-energy of $u$ by

$$
E_{p}(t)=\int_{E_{n}}\left\{\left|\frac{\partial u}{\partial t}(x, t)\right|^{p}+\left|\nabla_{x} u(x, t)\right|^{p}\right\} d x .
$$

Thus $E_{p}(0)=\|g\|_{p}^{p}+\|\nabla f\|_{p}^{p}$. The classical conservation of energy principle states that $E_{2}(t)$ is a constant. Littman [5] has shown that there is no analogue of this statement for $p \neq 2$; in fact there exist weak solutions with $E_{p}(0)<\infty$ and $E_{p}(t)=\infty$ for all $t \neq 0$.

Here we obtain estimates on the $L^{q}$ norm of $u(x, t)$ for fixed $t \neq 0$ in terms of the $p$-energy at time $t=0$ for certain values of $p$ and $q$. 
TheOREM 2. Let $u(x, t)$ be given by (16), $n \geqq 2$.

(a) For any $t \neq 0$

where

$$
\left(\int_{E_{n}}|u(x, t)|^{q} d x\right)^{1 / q} \leqq c(t) E_{p}(0)^{1 / p}
$$

and

$$
\frac{n}{q}=\frac{n-1}{2}-\frac{1}{p^{\prime}} \text { for } 2 \frac{n+1}{n+3} \leqq p \leqq 2
$$

( $p>1$ in case $n=2)$.

$$
\frac{1}{q}=\frac{n}{p}-\frac{n+1}{2} \text { for } 2 \frac{n}{n+2} \leqq p \leqq 2 \frac{n+1}{n+3}
$$

(b) For any $t \neq 0$ and any integer $k \geqq 1$

$$
\left\|u(\cdot, t): L_{k-1}^{q}\right\| \leqq c(t)\left(\left\|g: L_{k-1}^{p}\right\|+\left\|f: L_{k}^{p}\right\|\right)
$$

for $p$ and $q$ related as above. Here the norm

$$
\left\|f: L_{k}^{p}\right\|=\left(\sum_{|\alpha| \leqq k}\left\|\left(\frac{\partial}{\partial x}\right)^{\alpha} f\right\|_{p}^{p}\right)^{1 / p}
$$

REMARKS. The inequalities remain valid for any $f$ and $g$ for which the right side is finite. This follows by the usual limit argument.

The Sobolev inequalities imply

$$
\left\|f: L_{k-1}^{r}\right\| \leqq c\left\|f: L_{k}^{p}\right\| \quad \text { for } 1 / r=1 / p-1 / n, \quad 1<p, r<\infty .
$$

Since $u(x, 0)=f(x)$ we may compare this with our results for $t \neq 0$. We find that for the appropriate choice of $p$ (in fact $2 n /(n+2)<p<2$ ) we have $q>r$, i.e. $u(x, t)$ is better behaved at any time $t \neq 0$ than at time $t=0$.

We can use this remark to illustrate the phenomenon of focusing of singularities. Let $p=2(n+1) /(n+3)$ so that $q=2(n+1) /(n-1)$. We then choose $k$ so that $1 / q-(k-1) / n<0$ but $1 / p-k / n>0$. We can then find an $f \in L_{k}^{p}$ which is unbounded in any open set. Forming $u$ from $f$ with say $g=0$, we find that $u(\cdot, t)$ for any $t \neq 0$ is continuous and bounded by (b) and the Sobolev embedding theorem. Similarly we can construct weak solutions $u(x, t)$ which are continuously differentiable in $x$ for all $t \neq 0$ but not differentiable for $t=0$.

Proof of Theorem 2. We note that (b) follows from (a) since any space derivative $(\partial / \partial x)^{\alpha} u(x, t)$ is again a solution of the wave equation with Cauchy data $(\partial / \partial x)^{\alpha} f$ and $(\partial / \partial x)^{\alpha} g$.

To prove (a) we observe from (17) that it suffices to prove

$$
\left\|S_{(n-1) / 2} g\right\|_{q} \leqq c\|g\|_{p}
$$

and

$$
\left\|S_{(n+1) / 2} f\right\|_{q} \leqq c\|\nabla f\|_{p}
$$


Now (20) is just a special case of Theorem 1, and (21) is equivalent to

$$
\left\|\mathscr{F}^{-1}\left(\frac{\cos |\xi|}{|\xi|} \hat{f}(\xi)\right)\right\|_{q} \leqq c\|f\|_{p} \text {. }
$$

Let $\psi$ be a function in $C^{\infty}\left(E^{n}\right)$ which vanishes in a neighborhood of the origin and is identically one for large $\xi$. Then the Hardy-Littlewood multiplier theorem implies

$$
\left\|\mathscr{F}^{-1}(1-\psi(\xi)) \frac{\cos |\xi|}{|\xi|} \hat{f}(\xi)\right\|_{q} \leqq c\|f\|_{p}
$$

for the values of $p$ and $q$ given above since $1<p \leqq 2 \leqq q<\infty$ and $1 / p-1 / q \geqq 1 / n$.

Thus we must study the operator $\mathscr{F}^{-1}(\psi(\xi)(\cos |\xi| /|\xi|) \hat{f}(\xi))$. We introduce related analytic families defined by

$$
U_{\alpha}^{(j)}(f)=\mathscr{F}^{-1}\left(\psi(\xi) \xi_{j}|\xi|^{\alpha-n / 2-1} J_{n / 2-\alpha-1}(|\xi|) \hat{f}(\xi)\right), \quad j=1, \ldots, n,
$$

and imitate the proof of Theorem 1. Part (a) of Theorem 1 for $U_{\alpha}^{(j)}$ follows by exactly the same reasoning. To prove the analogue of (b) we must show for $\operatorname{Re}(\alpha)=0$ that

is in $L^{\infty}$ and

$$
\mathscr{F}^{-1}\left(|\xi|^{\alpha-n / 2-1} \xi_{j} \psi(\xi) J_{n / 2-\alpha-1}(|\xi|)\right)
$$

$$
\left\|\mathscr{F}^{-1}\left(|\xi|^{i b-n / 2-1} \psi(\xi) \xi_{j} J_{n / 2-1-i b}(|\xi|)\right)\right\|_{\infty} \leqq c e^{c|b|} .
$$

To prove this we begin with formula (4) for $\alpha=i b$ and deduce, using (7) and (8), that

$$
\begin{aligned}
\left(x_{j} \phi_{i b}\right)^{\wedge}(\xi)= & c(b)\left(\partial / \partial \xi_{j}\right)\left(|\xi|^{-n / 2-i b} J_{n / 2+i b}(|\xi|)\right) \\
= & c(b)\left(\xi_{j}|| \xi \mid\right)\left(-|\xi|^{-n / 2-i b} J_{n / 2+1+i b}(|\xi|)\right) \\
= & c(b) \xi_{j}|\xi|^{-n / 2-1-i b} J_{n / 2-1+i b}(|\xi|) \\
& -\xi_{j}|\xi|^{-n / 2-2-i b} J_{n / 2+i b}(|\xi|) c(b)(n+2 i b)
\end{aligned}
$$

hence

$$
\begin{aligned}
\mathscr{F}^{-1}\left(\psi(\xi) \xi_{j}|\xi|^{-n / 2-1-i b} J_{n / 2-1+i b}(|\xi|)\right)= & \frac{1}{c(b)} \mathscr{F}^{-1}\left(\psi(\xi)\left(x_{j} \phi_{i b}\right)^{\wedge}\right) \\
& +\frac{1}{(n+2 i b) c(b)} \mathscr{F}-1\left(\psi(\xi) \xi_{j}|\xi|^{-2} \hat{\phi}_{i b}\right) .
\end{aligned}
$$

Now $\psi(\xi)$ is the Fourier-Stieltjes transform of a bounded measure so the first term on the right is in $L^{\infty}$. The second term is in $L^{\infty}$ because the multiplier operator $\mathscr{F}^{-1}\left(\xi_{j}|\xi|^{-1} \hat{f}(\xi)\right)$ (the so-called $j$ th Riesz transform) is bounded on any $L^{p}, 1<p<\infty$, the operator $\mathscr{F}^{-1}\left(\psi(\xi)|\xi|^{-1} \hat{f}(\xi)\right)$ sends $L^{p}$ to $L^{\infty}$ for any $p>n$, and $\phi_{i b} \in L^{1} \cap L^{\infty}$. An examination of the various constants shows that the growth in $b$ is at most exponential.

Thus the same estimates hold for $U_{\alpha}^{(j)}$ as for $S_{\alpha}$. In particular setting $\alpha=(n-1) / 2$ we obtain

$$
\left\|\mathscr{F}^{-1}\left(\psi(\xi) \xi_{j}|\xi|^{-2} \cos |\xi| \hat{f}(\xi)\right)\right\|_{q} \leqq c\|f\|_{p}
$$


Applying the $j$ th Riesz transform and summing over $j$ we obtain

$$
\left\|\mathscr{F}-1\left(\psi(\xi) \frac{\cos |\xi|}{|\xi|} \hat{f}(\xi)\right)\right\|_{q} \leqq c\|f\|_{p}
$$

which, together with (23) gives the desired result.

Other applications of these ideas to the wave equation will be given elsewhere.

4. Spherical convolutions. In analogy with (2) we define for $n \geqq 2$

$$
\tilde{T}_{\alpha} f\left(x^{\prime}\right)=\int_{\Sigma} f\left(y^{\prime}\right)\left|x^{\prime} \cdot y^{\prime}\right|^{-\alpha} d y^{\prime}
$$

for $0<\alpha<1$ and $f$ defined on $\Sigma$. Later we will discuss the extension to $\alpha \geqq 1$.

THEOREM 3. (a) $\left\|\tilde{T}_{\alpha} f\right\|_{q} \leqq A_{\alpha}\|f\|_{p}$ provided $1<p \leqq 2 \leqq q<\infty$ and $1 / p-1 / q \leqq$ $(n-2 \alpha) /(2(n-1))$.

(b) $\left\|\tilde{T}_{\alpha} f\right\|_{p^{\prime}} \leqq A_{\alpha}\|f\|_{p}$ for $p=n /(n-\alpha), p^{\prime}=n / \alpha$.

(c) $\left\|\tilde{T}_{\alpha} f\right\|_{q} \leqq A_{\alpha}\|f\|_{p}$ for $\alpha \geqq \frac{1}{2}$ provided $n /(n-\alpha) \leqq p \leqq 2$ and $(n-1) / q=\alpha-1 / p^{\prime}$, or $(n-1) /\left(n-\frac{1}{2}-\alpha\right) \leqq p \leqq n /(n-\alpha)$ and $1 / q=\alpha-(n-1) / p^{\prime}$.

As one would expect, this is the direct analogue of Theorem 1 for $E^{n-1}$, since $\tilde{T}_{\alpha}$ is a spherical convolution with a kernel having a singularity on the $n-2$ dimensional equator of the $n-1$ sphere. However, there seems to be no simple way to deduce Theorem 3 from Theorem 1.

We shall give a proof which is quite different from the proof of Theorem 1 . We realize $\tilde{T}_{\alpha}$ as the real part of an operator $\tilde{S}_{\alpha}$ derived from the Fourier transform, and prove (a) and (b) for $\tilde{S}_{\alpha}$. We obtain (c) as before by interpolation.

We begin by deriving some properties of the Fourier transform.

Denote by $L^{p, \infty}\left(E^{n}\right)$ the space of functions $f(x)$ satisfying $m\{x:|f(x)|>s\}$ $\leqq M^{p} / s^{p}$ for all $s, 0<s<\infty$, and let $\|f\|_{p, \infty}^{*}$ be the least such $M$. It follows from Hunt's theorem [4] that the Fourier transform is a bounded operator from $L^{p, \infty}$ to $L^{p^{\prime}, \infty}$ for $1<p<2$ and $1 / p+1 / p^{\prime}=1$.

LEMMA 1. Let $f(x)=\Omega\left(x^{\prime}\right)|x|^{-n / p}, \quad 1<p<\infty$. Then $f \in L^{p, \infty}$ if and only if $\Omega \in L^{p}\left(S^{n-1}\right)$.

Proof. Note $|f(x)|>s$ if and only if $|x|<\left(\left|\Omega\left(x^{\prime}\right)\right| / s\right)^{p / n}$ so

$$
\begin{aligned}
m\{x:|f(x)|>s\} & =\int_{S^{n-1}} \int_{0}^{\left(\left|\Omega\left(x^{\prime}\right)\right| / s\right)^{p / n}} r^{n-1} d r d x^{\prime} \\
& =\frac{1}{n} \int_{S^{n-1}}\left(\frac{\left|\Omega\left(x^{\prime}\right)\right|}{s}\right)^{p} d x^{\prime} .
\end{aligned}
$$

Lemma 2. Let $1<p<2, \Omega \in L^{p}\left(S^{n-1}\right)$ and $f(x)=\Omega\left(x^{\prime}\right)|x|^{-n / p}$. Then the Fourier transform $\hat{f}(\xi)=\psi\left(\xi^{\prime}\right)|\xi|^{-n / p^{\prime}}$ for some $\psi \in L^{p^{\prime}}\left(S^{n-1}\right), 1 / p+1 / p^{\prime}=1$. 
Proof. By Lemma $1 f \in L^{p, \infty}$ hence $\hat{f} \in L^{p^{\prime}, \infty}$ by Hunt's theorem. Now

$$
\begin{aligned}
\hat{f}(\xi) & =\int \exp (i x \cdot \xi) f(x) d x=\int \exp \left(i x^{\prime} \cdot \xi^{\prime}|x||\xi|\right) \Omega\left(x^{\prime}\right)|x|^{-n / p} d x \\
& =|\xi|^{-n / p^{\prime}} \int \exp \left(i x \cdot \xi^{\prime}\right) \Omega\left(x^{\prime}\right)|x|^{-n / p} d x=|\xi|^{n / p^{\prime}} \hat{f}\left(\xi^{\prime}\right)
\end{aligned}
$$

where $\xi^{\prime}=\xi|| \xi \mid$. Thus $f$ has the desired form. Applying Lemma 1 again shows $\psi \in L^{p^{\prime}}$.

Definition. Let $\widetilde{S}_{p}, 1<p<2$, denote the map $\Omega \rightarrow \psi$ as in Lemma $2 ; S_{p} \Omega\left(\xi^{\prime}\right)$ $=\mathscr{F}\left(\Omega\left(x^{\prime}\right)|x|^{-n / p}\right)\left(\xi^{\prime}\right)$.

As an immediate corollary of Lemma 2, we have

$$
\left\|\widetilde{S}_{p} \Omega\right\|_{p^{\prime}}=A_{p}\|\Omega\|_{p} \quad \text { for all } \Omega \in L^{p}, \quad 1<p<2 .
$$

Lemma 3. Let $1<p<2$. Then

$$
\tilde{S}_{p} \Omega\left(\xi^{\prime}\right)=\lim _{k \rightarrow \infty} \int_{|x| \leqq N_{k}} \Omega\left(x^{\prime}\right)|x|^{-n / p} \exp \left(i x \cdot \xi^{\prime}\right) d x
$$

in the $L^{2}$ norm, for any sequence $N_{k} \rightarrow \infty$ sufficiently rapidly.

Proof. Since $f(x)=\Omega\left(x^{\prime}\right)|x|^{-n / p} \in L^{p, \infty}$ and $L^{p, \infty} \subset L^{1}+L^{2}$ we have

$$
\int_{|x| \leqq N} \Omega\left(x^{\prime}\right)|x|^{-n / p} \exp (i x \cdot \xi) d x
$$

converging to $\hat{f}(\xi)$ in the $L^{2}$ norm on the spherical shell $\frac{1}{2} \leqq|\xi| \leqq 2$. It follows that for subsequences $N_{k}^{\prime} \rightarrow \infty$ fast enough we must have convergence in $L^{2}$ on some sphere $|\xi|=a$ for $\frac{1}{2} \leqq a \leqq 2$. By homogeneity we obtain convergence on the sphere $|\xi|=1$ for the subsequences $a N_{k}^{\prime}$.

LEMMA 4. Let $1<p<n /(n-1)$ so that $0<n / p^{\prime}<1$. Let $\alpha=n / p^{\prime}$. Then

where

$$
\tilde{S}_{p} \Omega\left(\xi^{\prime}\right)=\int_{S^{n-1}} \Omega\left(x^{\prime}\right)\left(a_{p}+i b_{p} \operatorname{sgn}\left(x^{\prime} \cdot \xi^{\prime}\right)\right)\left|x^{\prime} \cdot \xi\right|^{-\alpha} d x^{\prime}
$$

$$
a_{p}=\lim _{N \rightarrow \infty} \int_{0}^{N} r^{\alpha-1} \cos r d r \text { and } b_{p}=\lim _{N \rightarrow \infty} \int_{0}^{N} r^{\alpha-1} \sin r d r
$$

for all $\Omega \in L^{p}$.

Proof. It follows by integration by parts not only that $a_{p}$ and $b_{p}$ exist and are finite, but also

$$
\sup _{N}\left|\int_{0}^{N} r^{\alpha-1} \cos r d r\right|=A_{p}<\infty \text { and } \sup _{N}\left|\int_{0}^{N} r^{\alpha-1} \sin r d r\right|=B_{p}<\infty
$$

for $0<\alpha<1$.

We compute

$$
\int_{|x| \leqq N} \Omega\left(x^{\prime}\right)|x|^{-n / p} \exp \left(i x \cdot \xi^{\prime}\right) d x=\int_{S^{n-1}} \Omega\left(x^{\prime}\right)\left[\int_{0}^{N} \exp \left(i r x^{\prime} \cdot \xi^{\prime}\right) r^{\alpha-1} d r\right] d x^{\prime} .
$$


Now

$$
\begin{aligned}
\int_{0}^{N} \exp \left(i r x^{\prime} \cdot \xi^{\prime}\right) r^{\alpha-1} d r & =\int_{0}^{N}\left(\cos r x^{\prime} \cdot \xi^{\prime}+i \sin r x^{\prime} \cdot \xi^{\prime}\right) r^{\alpha-1} d r \\
& =\left|x^{\prime} \cdot \xi^{\prime}\right|^{-\alpha} \int_{0}^{N\left|x^{\prime} \cdot \xi^{\prime}\right|}\left(r^{\alpha-1} \cos r+i \operatorname{sgn}\left(x^{\prime} \cdot \xi^{\prime}\right) r^{\alpha-1} \sin r\right) d r
\end{aligned}
$$

which converges to $\left|x^{\prime} \cdot \xi^{\prime}\right|^{-\alpha}\left(a_{p}+i b_{p} \operatorname{sgn}\left(x^{\prime} \cdot \xi^{\prime}\right)\right)$ if $x^{\prime} \cdot \xi^{\prime} \neq 0$. Now for almost every $\xi^{\prime} \in S^{n-1}$ we have $\left|x^{\prime} \cdot \xi^{\prime}\right|^{-\alpha}\left|\Omega\left(x^{\prime}\right)\right| \in L^{1}\left(S^{n-1}\right)$ so that the integrand

$$
\left|\Omega\left(x^{\prime}\right) \int_{0}^{N} \exp \left(i r x^{\prime} \cdot \xi^{\prime}\right) r^{\alpha-1} d r\right| \leqq\left(A_{p}+B_{p}\right)\left|\Omega\left(x^{\prime}\right)\right|\left|x^{\prime} \cdot \xi^{\prime}\right|^{-\alpha} \in L^{1}\left(S^{n-1}\right) .
$$

Thus we may apply the dominated convergence theorem to conclude

$$
\begin{aligned}
\lim _{N \rightarrow \infty} \int_{|x| \leqq N} \Omega\left(x^{\prime}\right)|x|^{-n / p} \exp \left(i x \cdot \xi^{\prime}\right) d x \\
=\int_{S^{n-1}} \Omega\left(x^{\prime}\right)\left(a_{p}+i b_{p} \operatorname{sgn}\left(x^{\prime} \cdot \xi^{\prime}\right)\right)\left|x^{\prime} \cdot \xi^{\prime}\right|^{-\alpha} d x^{\prime}
\end{aligned}
$$

for almost every $y^{\prime} \in S^{n-1}$.

Lemma 4 shows that $\tilde{T}_{\alpha} \Omega=\operatorname{Re} \tilde{S}_{p} \Omega$ for real valued $\Omega$ and $\alpha=n / p^{\prime}, 0<\alpha<1$. Thus (30) established part (b) of Theorem 3. To establish part (a) we expand $\Omega$ in spherical harmonics and compute $S_{p}$ in terms of the expansion.

Let $Y_{k}(x)$ be any harmonic polynomial homogeneous of degree $k$. We use the formula [2], [11]

$$
\mathscr{F}\left(|x|^{\alpha-n-k} Y_{k}(x)\right)=\gamma_{k, \alpha} Y_{k}(\xi)|\xi|^{-k-\alpha},
$$

where

$$
\gamma_{k, \alpha}=\pi^{-\alpha+n / 2} i^{k} \Gamma(\alpha / 2+k / 2) / \Gamma(k / 2+n / 2-\alpha / 2) .
$$

Thus if $\Omega=\sum_{k=0}^{N} Y_{k}\left(x^{\prime}\right)$ we have

$$
\tilde{S}_{p} \Omega=\sum_{k=0}^{N} \gamma_{k, \alpha} Y_{k}\left(x^{\prime}\right), \quad \text { where } \alpha=\frac{n}{p^{\prime}},
$$

and this uniquely determines $\tilde{S}_{p}$. We can study the properties of such a spherical harmonic multiplier transform using a result of Marcinkiewicz and Zygmund [6].

Let $\Omega$ be any sufficiently regular function on $\Sigma$. Then $\Omega$ has a spherical harmonic expansion $\Omega=\sum_{k=0}^{\infty} a_{k} Y_{k}\left(x^{\prime}\right)$ where the $Y_{k}$ are spherical harmonics of degree $k$ normalized so that $\int_{\Sigma}\left|Y_{k}\left(x^{\prime}\right)\right|^{2} d x=1$. For different choices of $\Omega$ we must choose different $Y_{k}$, but this will not matter. The system $\left\{Y_{k}\right\}$ is orthonormal and satisfies

$$
\left\|Y_{k}\right\|_{\infty} \leqq\left(\operatorname{dim} H_{k}\right)^{1 / 2} \leqq M k^{(n-2) / 2},
$$

where $H_{k}$ is the space of all spherical harmonics of degree $k$. Thus by Theorems 1 , $2,3,4$ and the final remark of [6],

$$
\|\Omega\|_{q} \leqq A\left(\sum_{k=0}^{\infty}\left|a_{k}\right|^{r}(1+k)^{(n / 2-(n-1) / q) r-1}\right)^{1 / r} \text { for } 2 \leqq q<\infty, q^{\prime} \leqq r \leqq q,
$$


and

(36) $\left(\sum_{k=0}^{\infty}\left|a_{k}\right|^{r}(1+k)^{(n / 2-(n-1) / p) r-1}\right)^{1 / r} \leqq A\|\Omega\|_{p} \quad$ for $1<p \leqq 2, p \leqq r \leqq p^{\prime}$.

LEMMA 5. Let $c_{k}$ be any sequence of complex numbers satisfying $\left|c_{k}\right| \leqq B(k+1)^{-\beta}$. If $S \Omega\left(x^{\prime}\right)=\sum_{k=0}^{\infty} a_{k} c_{k} Y_{k}\left(x^{\prime}\right)$ then $\|S \Omega\|_{q} \leqq A^{2} B\|\Omega\|_{p}$ provided $1<p \leqq 2 \leqq q<\infty$ and $1 / p-1 / q=\beta /(n-1)$.

Proof. We imitate the proof of Hardy-Littlewood for Fourier series. Using (35) and (36) with $r=2$, we have

$$
\begin{aligned}
\|S \Omega\|_{q} & \leqq A B\left(\sum_{k=0}^{\infty}\left|a_{k}\right|^{2}(1+k)^{-2 \beta+n-2(n-1) / q-1}\right)^{1 / 2} \\
& =A B\left(\sum_{k=0}^{\infty}\left|a_{k}\right|^{2}(1+k)^{n-2(n-1) / p-1}\right)^{1 / 2} \leqq A^{2} B\|\Omega\|_{p} .
\end{aligned}
$$

It is now a simple matter to deduce part (a) of Theorem 3 for $\widetilde{S}_{n /(n-\alpha)}$ in place of $\tilde{T}_{\alpha}$ for $0<\alpha<n / 2$. For by (32) and Stirling's formula

$$
\left|\gamma_{k, \alpha}\right| \leqq c_{\alpha}(1+k)^{\alpha-n / 2}
$$

so Lemma 5 applies with $\beta=n / 2-\alpha$.

This completes the proof of Theorem 3. It is possible to extend definition (29) to nonintegral $\alpha>1$ by formally integrating by parts along great circles through $x^{\prime}$. It is also possible to extend Lemma 4 to the range $0<\alpha<n / 2, \alpha$ nonintegral, with some slight modifications when $n=3$ due to boundary terms. Theorem 3 will continue to hold.

\section{REFERENCES}

1. I. M. Gel'fand and G. E. Silov, Generalized functions. Vol. I: Operations on them, Fizmatgiz, Moscow, 1958; English transl., Academic Press, New York, 1964. MR 20 \#4182; MR 29 \#3869.

2. C. Herz, On the mean inversion of Fourier and Hankel transforms, Proc. Nat. Acad. Sci. U.S.A. 40 (1954), 996-999. MR 16, 127.

3. L. Hörmander, Estimates for translation invariant operators in $L^{p}$ spaces, Acta Math. 104 (1960), 93-140. MR 22 \#12389.

4. R. Hunt, An extension of the Marcinkiewicz interpolation theorem to Lorentz spaces, Bull. Amer. Math. Soc. 70 (1964), 803-807; Addendum, 71 (1965), 396. MR 29 \#6292.

5. W. Littman, The wave operator and $L_{p}$ norms, J. Math. Mech. 12 (1963), 55-68. MR 26 \#4043.

6. J. Marcinkiewicz and A. Zygmund, Some theorems on orthogonal systems, Fund. Math. 28 (1937), 309-335.

7. E. M. Stein, Interpolation of linear operators, Trans. Amer. Math. Soc. 83 (1956), 482-492. MR 18, 575.

8. - Localization and summability of multiple Fourier series, Acta Math. 100 (1958), 93-147. MR 21 \#4331. 
9. G. N. Watson, A treatise on the theory of Bessel functions, 2nd ed., Cambridge Univ. Press, Cambridge and Macmillan, New York, 1944. MR 6, 64.

10. A. Zygmund, Trigonometric series, 2nd rev. ed., Cambridge Univ. Press, New York, 1959. MR 21 \#6498.

11. S. Bochner, Theta relations with spherical harmonics, Proc. Nat. Acad. Sci. U.S.A. 37 (1951), 804-808. MR 14, 43.

Massachusetts Institute of TeChNology,

CAMbridge, MassachusetTs 02138 